\title{
Improvement in PET/CT Image Quality with a Combination of Point-Spread Function and Time-of-Flight in Relation to Reconstruction Parameters
}

\author{
Go Akamatsu ${ }^{1}$, Kaori Ishikawa ${ }^{1}$, Katsuhiko Mitsumoto ${ }^{1}$, Takafumi Taniguchi ${ }^{1}$, Nobuyoshi Ohya ${ }^{2}$, Shingo Baba ${ }^{3}$, \\ Koichiro $\mathrm{Abe}^{3}$, and Masayuki Sasaki ${ }^{1}$ \\ ${ }^{1}$ Department of Health Sciences, Graduate School of Medical Sciences, Kyushu University, Fukuoka, Japan; ${ }^{2}$ Department of Medical \\ Technology, Kyushu University Hospital, Fukuoka, Japan; and ${ }^{3}$ Department of Clinical Radiology, Graduate School of Medical \\ Sciences, Kyushu University, Fukuoka, Japan
}

The aim of this study was to investigate the effects of the pointspread function (PSF) and time-of-flight (TOF) on improving ${ }^{18} \mathrm{~F}$ FDG PET/CT images in relation to reconstruction parameters and noise-equivalent counts (NEC). Methods: This study consisted of a phantom study and a retrospective analysis of 39 consecutive patients who underwent clinical ${ }^{18} \mathrm{~F}-\mathrm{FDG}$ PET/CT. The body phantom of the National Electrical Manufacturers Association and International Electrotechnical Commission with a 10-mm-diameter sphere was filled with an ${ }^{18} \mathrm{~F}$-FDG solution with a $4: 1$ radioactivity ratio compared with the background. The PET data were reconstructed with the baseline orderedsubsets expectation maximization (OSEM) algorithm, with the OSEM + PSF model, with the OSEM + TOF model, and with the OSEM+PSF+TOF model. We evaluated image quality by visual assessment, the signal-to-noise ratio of the $10-\mathrm{mm}$ sphere $\left(\mathrm{SNR}_{10 \mathrm{~mm}}\right)$, the contrast of the $10-\mathrm{mm}$ sphere, and the coefficient of variance in the phantom study and then determined the optimal reconstruction parameters. We also examined the effects of PSF and TOF on the quality of clinical images using the signal-to-noise ratio in the liver $\left(\mathrm{SNR}_{\text {liver }}\right)$ in relation to the NEC in the liver $\left(\mathrm{NEC}_{\text {liver }}\right)$. Results: In the phantom study, the $\mathrm{SNR}_{10} \mathrm{~mm}$ was the highest for the OSEM+PSF+TOF model, and the highest value was obtained at iteration 2 for algorithms with the TOF and at iteration 3 for those without the TOF. In terms of a postsmoothing filter full width at half maximum (FWHM), the high $\mathrm{SNR}_{10} \mathrm{~mm}$ was obtained with no filtering or was smaller than $2 \mathrm{~mm}$ for algorithms with PSF and was 4-6 $\mathrm{mm}$ for those without PSF. The balance between the contrast recovery and noise is different for algorithms with either PSF or TOF. A combination of PSF and TOF improved $\mathrm{SNR}_{10 \mathrm{~mm}}$, contrast, and coefficient of variance, especially with a small-FWHM gaussian filter. In the clinical study, the $\mathrm{SNR}_{\text {liver }}$ of the low$\mathrm{NEC}_{\text {liver }}$ group in the OSEM+PSF+TOF model was compared

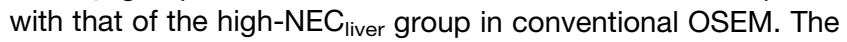
PSF+TOF improved the $\mathrm{SNR}_{\text {liver }}$ by about $24.9 \% \pm 9.81 \%$. Conclusion: A combination of PSF and TOF clearly improves

Received Jan. 30, 2012; revision accepted Jun. 18, 2012.

For correspondence or reprints contact: Masayuki Sasaki, Division of Medical Quantum Science, Department of Health Sciences, Graduate School of Medical Sciences, Kyushu University, 3-1-1 Maidashi, Higashi-ku, Fukuoka 812-8582, Japan.

E-mail: msasaki@hs.med.kyushu-u.ac.jp

Published online Sep. 4, 2012.

COPYRIGHT @ 2012 by the Society of Nuclear Medicine and Molecular Imaging, Inc. image quality, whereas optimization of the reconstruction parameters is necessary to obtain the best performance for PSF or TOF. Furthermore, this combination has the potential to provide good image quality with either lower activity or shorter acquisition time, thus improving patient comfort and reducing the radiation burden.

Key Words: point-spread function; time-of-flight; PET/CT; image quality; noise-equivalent counts

J Nucl Med 2012; 53:1716-1722

DOI: 10.2967/jnumed.112.103861

$\mathbf{P}$ ET/CT with ${ }^{18} \mathrm{~F}-\mathrm{FDG}$ is useful for the detection and staging of various malignant tumors, monitoring of their response to therapy, and prognostic stratification (1-6). High image quality is required for an accurate diagnosis, although the spatial resolution of PET is relatively low in comparison to other imaging modalities (7). Recently, the information provided by the point-spread function (PSF) and time-of-flight (TOF) has been expected to improve the spatial resolution and signal-to-noise ratio (SNR), respectively, of PET images (8-10).

A PET scanner consists of a circular ring of detectors. This geometry introduces distortion into the detection process. When the photon comes from the center of the field of view (FOV), the line of response is likely to be correctly localized in the crystal into which the photon entered. However, the farther the photon is from the center of the FOV, the more likely it is that the line of response might be incorrectly calculated, because the photon passes through the crystal at an angle and continues traveling to another crystal, resulting in increased distortion at points farther from the center of the FOV. To compensate for this geometric distortion, PSFs were measured at several million points in the FOV using a point source. The measured PSFs were then incorporated into the estimation step of the reconstruction algorithm. The modeling of the PSF reconstruction effectively positions the line of responses in their actual geometric location. PSF has been 
shown to improve both the spatial resolution and noise properties of reconstructed PET images (11).

The idea of using TOF information was first proposed in the 1960s $(12,13)$. The use of scintillators with a fast decay time was necessary to construct the TOF PET system, although those available at that time, such as cesium fluoride and barium fluoride, were of low density (14). High-density bismuth germanate has been widely used to pursue a highsensitivity system, although its slow decay time has not been suitable for the TOF strategy. The development of lutetium orthosilicate and lutetium yttrium orthosilicate has reawakened interest in TOF PET (15), because these scintillators have both a fast decay time and high density $(16,17)$. The TOF information provides a difference in arrival times between a pair of coincident photons and narrows the probability that the annihilation event will be located along the line of response. This additional information enables the reconstruction algorithm to converge to the expected image with fewer iterations and a better noise level (18).

PSF and TOF both have the potential to improve image quality and lesion detectability (19). Recent studies have reported faster convergence of lesion contrast and improvements in lesion detection performance with TOF (18-22). However, the effects of PSF and TOF have not been sufficiently demonstrated in different reconstruction parameters or investigated for their effects on noise levels. In this study, we determined the optimal reconstruction parameters to confirm the effects of PSF and TOF in a phantom study. Then, we examined the effects of PSF and TOF on the quality of clinical images.

\section{MATERIALS AND METHODS}

\section{Data Acquisition and Image Reconstruction}

We used a Biograph mCT scanner (Siemens Healthcare) for all PET acquisitions in this study. This PET scanner comprises 3 rings with a total of 144 lutetium orthosilicate detectors, covering an axial FOV of $16.2 \mathrm{~cm}$ and a transaxial FOV of $70 \mathrm{~cm}$ in diameter; each block is $4 \times 4 \times 20 \mathrm{~mm}$. The coincidence time window was $4.1 \mathrm{~ns}$. The TOF time resolution was $555 \mathrm{ps}$. The PET data were acquired in 3-dimensional mode and were reconstructed with the baseline ordered-subsets expectation maximization (OSEM) algorithm, with the OSEM+PSF model, with the OSEM+TOF model, and with the OSEM+PSF+TOF model. The default reconstruction parameters for OSEM and OSEM+PSF were 2 iterations and 24 subsets, and those for OSEM+TOF and OSEM+PSF+TOF were 2 iterations and 21 subsets. A gaussian filter with a full width at half maximum (FWHM) of $4 \mathrm{~mm}$ was used as a postsmoothing filter in all reconstruction models. The image matrix was $256 \times$ 256, with 3.18-mm pixels. The PET image slice thickness was $5 \mathrm{~mm}$. In a phantom study, we varied the number of iterations and the FWHM of the gaussian filter in all reconstruction models. A whole-body CT scan protocol was performed using the following parameters: $120 \mathrm{kV}, 100 \mathrm{~mA}, 0.5$-s tube rotation, and 5-mm slice collimation. The CT data were used for attenuation correction.

\section{Phantom Studies}

Imaging Protocol. Phantom study 1 was performed to determine an appropriate scanning time to enable visualization of a 10-mm-diameter hot sphere of unknown localization. The experiments were performed according to the "Japanese Guideline for Oncology FDG PET/CT Data Acquisition Protocol" (23). We used a body phantom of the National Electrical Manufacturers Association and International Electrotechnical Commission. Only one 10-mm-diameter sphere was embedded in the phantom and filled with an ${ }^{18} \mathrm{~F}$-FDG solution that had a 4:1 radioactivity ratio compared with the background. The background activity level of $5.31 \mathrm{kBq} / \mathrm{mL}$ was chosen to simulate liver uptake (24). The PET data were acquired in 3-dimensional list mode for $10 \mathrm{~min}$. PET images of different acquisition times ranging from 1 to $10 \mathrm{~min}$ were reconstructed and evaluated for the detectability of the hot sphere.

In our second phantom study, the PET data from a 2-min acquisition were reconstructed using various parameters, with iterations ranging from 1 to 10 and the gaussian filter having a FWHM ranging from 1 to $10 \mathrm{~mm}$. We determined the optimal reconstruction parameters to examine the effect of the reconstruction algorithm on the clinical images according to the results of this phantom study.

Data Analysis. The phantom PET image analyses were performed by visual assessment and by examining the SNR, the contrast of a 10-mm hot sphere, and the coefficient of variance on the background $(\mathrm{CV})$. We placed a circular region of interest (ROI) $10 \mathrm{~mm}$ in diameter on the $10-\mathrm{mm}$ hot sphere. We also placed a large circular ROI $100 \mathrm{~mm}$ in diameter on the slice of the sphere center and on slices $\pm 1 \mathrm{~cm}$ and $\pm 2 \mathrm{~cm}$ away from the background (total of 5 ROIs).

For visual assessment, the PET images were evaluated according to the detectability of the 10-mm-diameter hot sphere on a 3step scale ( 0 , not visualized; 1 , visualized, but similar hot spots were observed elsewhere; and 2, identifiable) by 1 board-certified nuclear medicine physician and 2 radiologic technologists. These investigators were unaware of the localization or the slice number on which the hot sphere was to be visualized. A score of 1.5 is considered to represent a clinically interpretable study (21).

The SNR of a 10 -mm-diameter hot sphere $\left(\mathrm{SNR}_{10 \mathrm{~mm}}\right)$, contrast, and CV were used as parameters for the physical assessment of image quality. The $\mathrm{SNR}_{10} \mathrm{~mm}$ was calculated as follows:

$$
\begin{aligned}
\mathrm{SNR}_{10 \mathrm{~mm}} & =[\text { signal }(\text { maximum value }) \\
& - \text { background }(\text { mean value })] / \mathrm{SD},
\end{aligned}
$$

where the signal was the maximum activity in the $10-\mathrm{mm}$ hot sphere ROI, the background was the mean activity in the 5 background ROIs, and SD was the mean of the SD of the 5 background ROI counts. The contrast of the 10-mm hot sphere and the CV were, respectively, calculated as follows:

$$
\begin{gathered}
\text { Contrast }=\operatorname{signal}(\text { maximum value }) / \text { background }(\text { mean value }) \\
\qquad \mathrm{CV}=\mathrm{SD} / \text { background }(\text { mean value }) \times 100(\%)
\end{gathered}
$$

\section{Clinical Study}

Imaging Protocol. A total of 39 consecutive patients (mean age, $63.5 \pm 14.3$ y) who underwent ${ }^{18}$ F-FDG PET/CT between March 24 and April 16, 2010, were analyzed in this study. Patients with liver lesions or with a fasting blood sugar level higher than 120 $\mathrm{mg} / \mathrm{dL}$ were excluded. The clinical indication for the ${ }^{18} \mathrm{~F}-\mathrm{FDG}$ PET/CT examinations of these patients were the evaluation of lung 
cancer $(n=8)$, malignant lymphoma $(n=6)$, thyroid cancer $(n=$ $6)$, general cancer screening $(n=5)$, colorectal cancer $(n=4)$, head and neck cancer $(n=4)$, cervical cancer $(n=2)$, prostate cancer $(n=1)$, malignant mesothelioma $(n=1)$, gastric cancer $(n=1)$, and unknown primary cancer $(n=1)$. The patients had an average weight of $59.9 \pm 14.2 \mathrm{~kg}$ (range, $35-89 \mathrm{~kg}$ ); their height averaged $160.7 \pm 8.4 \mathrm{~cm}$ (range, $145-176 \mathrm{~cm}$ ); and their body mass index (BMI) averaged $23.0 \pm 4.3 \mathrm{~kg} / \mathrm{m}^{2}$ (range, 16.0-31.6 $\mathrm{kg} / \mathrm{m}^{2}$ ). This clinical study was approved by the ethics committee of our institution (no. 22-48). This study was retrospective, and its results did not influence any further therapeutic decision making.

All patients fasted for at least $4 \mathrm{~h}$ before PET/CT and rested for $1 \mathrm{~h}$ after receiving an intravenous injection of $223.4 \pm 49.3 \mathrm{MBq}$ (range, 135.9-314.1 MBq) of ${ }^{18}$ F-FDG. The injected dose per kilogram averaged $4.0 \pm 1.4 \mathrm{MBq} / \mathrm{kg}$ (range, $1.83-7.81 \mathrm{MBq} /$ $\mathrm{kg}$ ). Scanning started at $1 \mathrm{~h}$ after administration and was performed from the mid thigh to the top of the head. The clinical PET data were acquired in the 3-dimensional static mode for 2 min per bed position. The bed overlap was $50 \%$. The PET/CT images were reconstructed with optimal parameters for each reconstruction algorithm according to the results of the phantom study.

Data Analysis. The noise-equivalent count (NEC) is a raw data quality metric that does not take into account the impact of reconstruction algorithms or of spatial resolution effects (25). To evaluate the quality of the acquired PET data, the NEC in the liver $\left(\mathrm{NEC}_{\text {liver }}\right)$ was calculated as follows:

$$
\mathrm{NEC}_{\text {liver }}=(1-\mathrm{SF})^{2} \times(\mathrm{T}+\mathrm{S})^{2} /(\mathrm{T}+\mathrm{S}+\mathrm{kR}),
$$

where $\mathrm{T}$ is the true coincidence counts, $\mathrm{S}$ is the scatter coincidence counts, $\mathrm{R}$ is the random coincidence counts, SF is the scatter fraction, and $\mathrm{k}=2$ (noise random correction factor). The true, scatter, and random coincidence counts were obtained from the full sinogram data of the bed position that covered the liver in the whole-body scan.

The quality of the clinical PET image was analyzed using the $\mathrm{SNR}$ in the liver $\left(\mathrm{SNR}_{\text {liver }}\right)$. We placed 3 circular ROIs in a $3-\mathrm{cm}$ diameter on 3 axial slices around the largest liver section; thus, a total 9 ROIs were placed. The SNR in the liver was calculated as follows:

$$
\mathrm{SNR}_{\text {liver }}=\mathrm{C}_{\text {liver }} / \mathrm{SD}_{\text {liver }}
$$

where $\mathrm{C}_{\text {liver }}$ was the mean count in the 9 liver ROIs, and $\mathrm{SD}_{\text {liver }}$ was defined as the SD of the 9 liver ROIs.

\section{Statistical Analysis}

The $\mathrm{SNR}_{\text {liver }}$ values were compared using the Wilcoxon signedranks test. In all analyses, a $P$ value of less than 0.05 was considered to be statistically significant.

\section{RESULTS}

\section{Phantom Studies}

The 10-mm hot sphere could be recognized in all PET images (Fig. 1A). In PET images from the 1-min acquisition reconstructed by both OSEM and OSEM+PSF, the hot sphere was relatively unclear. However, the hot sphere was clearly observed by reconstruction with TOF. In terms of the uniformity of the background, OSEM+PSF and OSEM+PSF+TOF were superior to conventional OSEM. Figure 1B shows the results of the visual assessment of the PET images according to each reconstruction model. The PSF and TOF improved the image quality, especially for images with a short acquisition time. The recommended image quality score of 1.5 is achieved with a 2-min acquisition reconstructed with OSEM+PSF+TOF, whereas longer scans were required when using other reconstruction algorithms.

The PET images reconstructed by various iteration numbers are shown in Figure 2A. Although the $10-\mathrm{mm}$ hot sphere became clearer with increasing iteration number, the uniformity of the background deteriorated. The image quality for the various numbers of iterations is shown in Figure $2 \mathrm{~B}$. The $\mathrm{SNR}_{10 \mathrm{~mm}}$, contrast, and $\mathrm{CV}$ are plotted in this figure as a function of the iteration number. The contrast is also plotted as a function of the $\mathrm{CV}$, and each plot
FIGURE 1. PET images and visual scores for different acquisition times (iteration 2, subsets 21 or 24). (A) Image quality of OSEM+PSF and OSEM+PSF+TOF models was superior to that of conventional OSEM, especially for short acquisition time. (B) Visual score of OSEM+PSF+TOF model for acquisition time of 2 min or longer yielded recommended value of 1.5 .

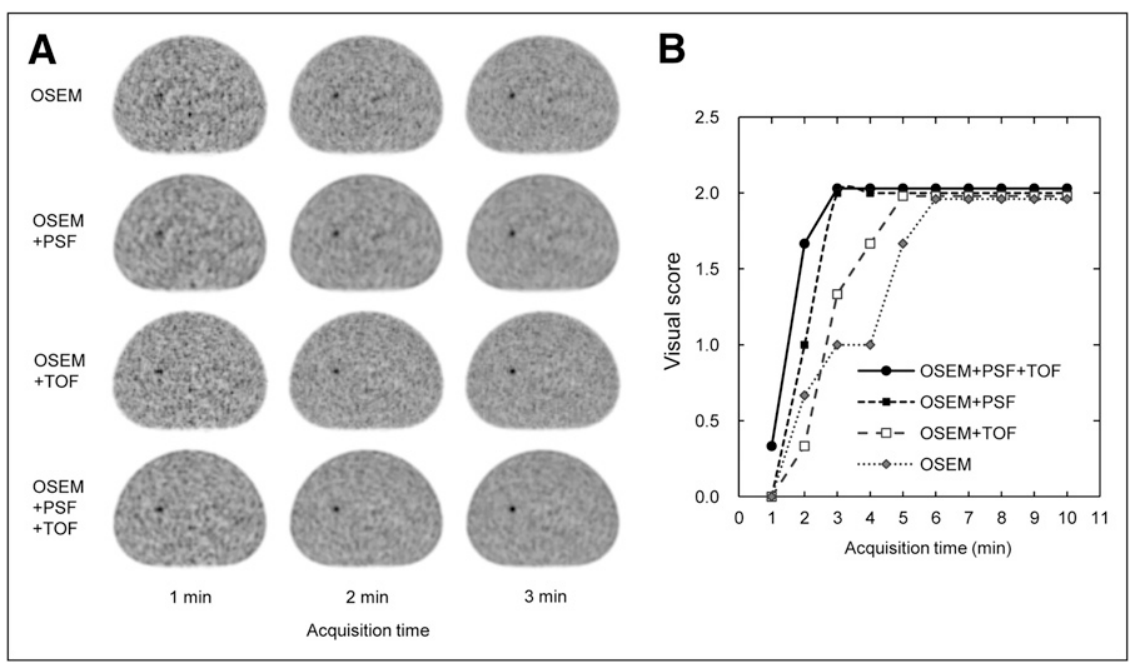



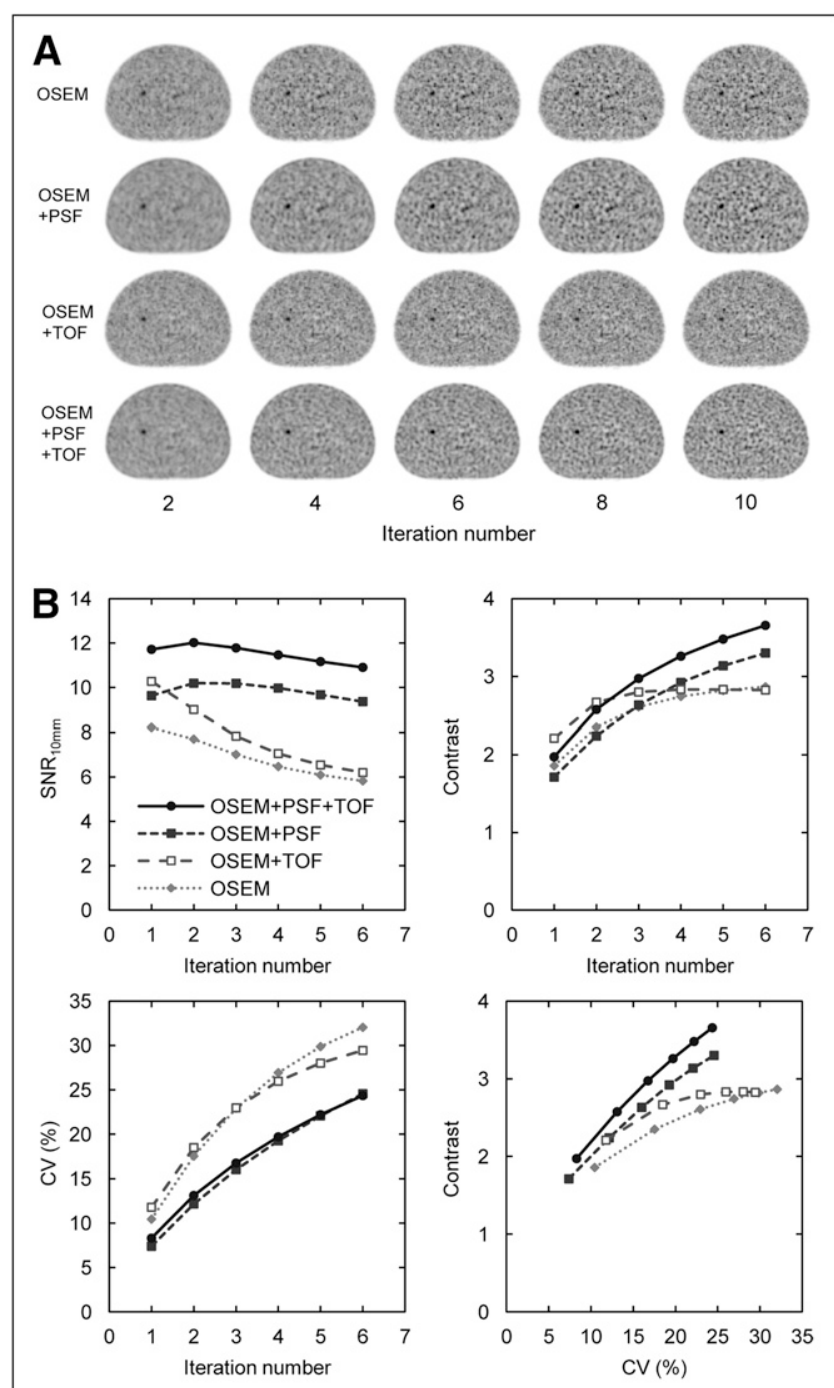

FIGURE 2. PET images for 2-min acquisitions and image quality for various numbers of iterations. (A) PET images reconstructed by OSEM, OSEM+PSF, OSEM+TOF, and OSEM + PSF+TOF in relation to iteration number. (B) $\mathrm{SNR}_{10} \mathrm{~mm}$, contrast, and $\mathrm{CV}$ in OSEM+PSF+TOF were superior to those obtained using other algorithms. $\mathrm{SNR}_{10 \mathrm{~mm}}$ for OSEM+PSF+TOF model was highest at iteration 2.

corresponds to the iteration number. The $\mathrm{SNR}_{10} \mathrm{~mm}$ and contrast were improved by TOF correction. In the algorithms with the PSF correction (OSEM+PSF and $\mathrm{OSEM}+\mathrm{PSF}+\mathrm{TOF}$ ), the $\mathrm{SNR}_{10 \mathrm{~mm}}$, contrast, and CV were generally superior to those obtained by the algorithms without the PSF correction. The $\mathrm{SNR}_{10} \mathrm{~mm}$ was the highest for the OSEM+PSF+TOF model, and the highest value was obtained at iteration 2 for OSEM+PSF+TOF and at iteration 3 for OSEM+PSF. The contrast for the $10-\mathrm{mm}$ sphere using OSEM+TOF reached a maximum and converged uniformly with smaller iteration numbers in comparison with the data obtained using other algorithms. When the PSF was included, the iterative convergence of the contrast was delayed and the $\mathrm{CV}$ was improved. The relationship between the $\mathrm{CV}$ and contrast showed that $\mathrm{OSEM}+\mathrm{PSF}+\mathrm{TOF}$ at iteration 2 yielded contrast similar to what was obtained with OSEM+PSF at iteration 3, but the TOF image had less noise. On the other hand, both OSEM+PSF+TOF and OSEM+PSF at iteration 2 yielded comparable noise levels, with the only disadvantage being a small loss of contrast for OSEM+PSF. On the basis of these results, an iteration number of 2 for algorithms with TOF and 3 for those without TOF was recommended.

The PET images reconstructed using various FWHMs of the gaussian filter are shown in Figure 3A. The hot sphere became more blurred with the increase in the filter FWHM width, whereas the background became homogeneous. Both the $\mathrm{SNR}_{10} \mathrm{~mm}$ and $\mathrm{CV}$ obtained by the algorithms with PSF were superior to those obtained by other algorithms (Fig. 3B). The high $\mathrm{SNR}_{10} \mathrm{~mm}$ was obtained with no filtering or with filtering less than $2 \mathrm{~mm}$ for OSEM+PSF and OSEM+PSF+TOF and 4-6 $\mathrm{mm}$ for OSEM and OSEM+TOF. The contrast decreased with increasing FWHM in all algorithms. The contrast was higher for OSEM+TOF using a small-FWHM gaussian filter. On the other hand, the CV was superior for OSEM+PSF. Both PSF and TOF improved the $\mathrm{SNR}_{10 \mathrm{~mm}}$, contrast, and CV, especially with a small-FWHM gaussian filter. Therefore, the optimal FWHM of the gaussian filter was recommended to be no filtering or less than $2 \mathrm{~mm}$ for algorithms with PSF and 4-6 mm for those without PSF. We determined

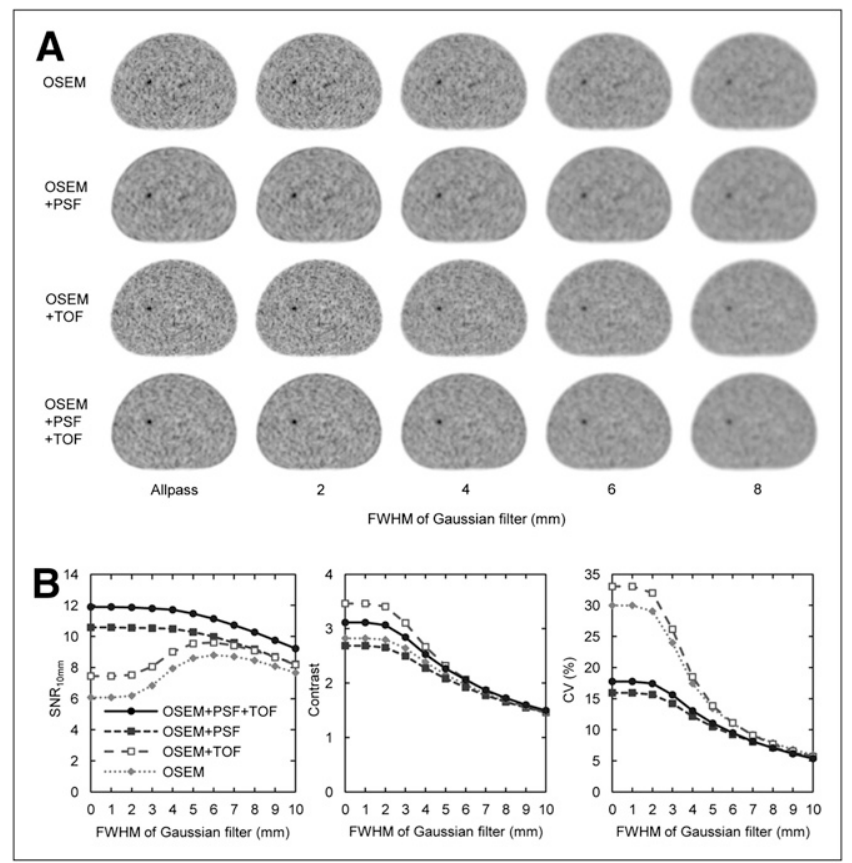

FIGURE 3. PET images at various FWHMs of gaussian filter. (A) PET images reconstructed by OSEM, OSEM+PSF, OSEM+TOF, and OSEM+PSF+TOF in relation to FWHM. (B) PSF and TOF improved SNR, contrast, and CV, especially for small-FWHM gaussian filter. 
that $2 \mathrm{~mm}$ for algorithms with PSF and $4 \mathrm{~mm}$ for those without PSF were the most appropriate FWHM widths.

\section{Clinical Studies}

The mean and SD of the $\mathrm{NEC}_{\text {liver }}$ was $3.90 \pm 0.80$ megacounts (range, 1.71-4.77 megacounts). The patients were classified into 2 groups based on a $\mathrm{NEC}_{\text {liver }}$ of 3.0 mega-

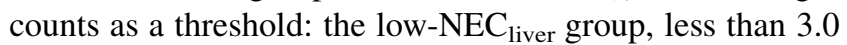
megacounts (average, 2.46 megacounts, $n=20$ ), and the high-NEC $\mathrm{N}_{\text {liver }}$ group, 3.0 or more megacounts (average, 3.72 megacounts, $n=19$ ). Figure 4 shows the $\mathrm{SNR}_{\text {liver }}$. In all patients, the $\mathrm{SNR}_{\text {liver }}$ of OSEM+TOF and OSEM+PSF+TOF was superior to that of the baseline OSEM $(P<0.01)$. Although the filter FWHM width differed, the $\mathrm{SNR}_{\text {liver }}$ of OSEM + PSF was equivalent to that of conventional OSEM. Concerning the relationship between the $\mathrm{SNR}_{\text {liver }}$ and $\mathrm{NEC}_{\text {liver, }}$, the $\mathrm{SNR}_{\text {liver }}$ of the high-NEC $\mathrm{N}_{\text {liver }}$ group was higher than that of the low-NEC $\mathrm{N}_{\text {liver }}$ group. The TOF significantly improved the $\mathrm{SNR}_{\text {liver }}$ in both groups $(P<0.05)$. The PSF+TOF improved the $\mathrm{SNR}_{\text {liver }}$ by $24.9 \% \pm 9.81 \%$. The $\mathrm{SNR}_{\text {liver }}$ improvement in both the OSEM+TOF model and the OSEM+PSF+TOF model did not correlate with $\mathrm{NEC}_{\text {liver }}$.

Figure 5 shows PET images of a representative patient. The PSF and TOF clearly improved the uniformity of the liver and the sharpness of the edge of the vertebral body. In addition, the activity of the physiologically low-uptake

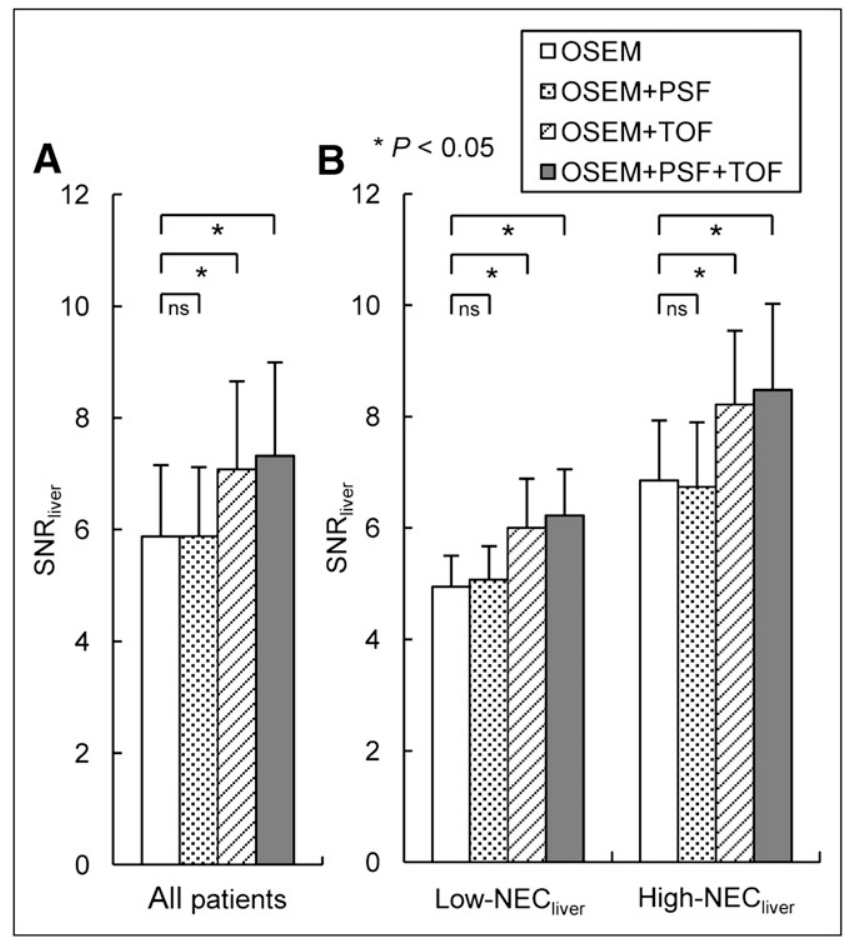

FIGURE 4. Relationship between SNR in liver and reconstruction algorithm in all patients (A) and in different NEC groups (B). SNR liver of algorithms with TOF were superior to those without TOF. SNR liver $_{\text {iv }}$

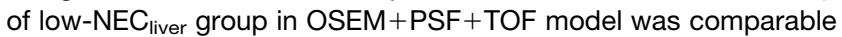
to that of high-NEC $\mathrm{N}_{\text {liver }}$ group in conventional OSEM.

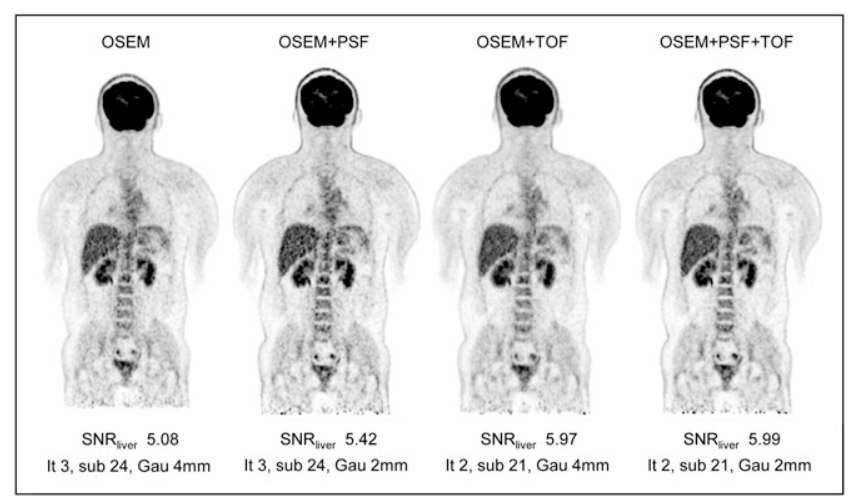

FIGURE 5. Patient images reconstructed by different algorithms. Patient with postsurgical state of colorectal cancer (BMI, 25.2; body weight, $71 \mathrm{~kg}$; injected dose, $296.8 \mathrm{MBq}$; NEC liver, 2.91 megacounts). It, sub, and Gau represent iteration, subsets, and gaussian filter FWHM width, respectively. TOF clearly improved uniformity of liver and sharpness of edge of vertebral body. Uptake in physiologically low-uptake areas, such as perirenal fat, lung, and intervertebral disks, was decreased by TOF correction. Combination of PSF and TOF markedly improved image quality.

areas, such as the perirenal fat, lungs, and intervertebral disks, decreased clearly.

\section{DISCUSSION}

In this study, we evaluated the effects of both PSF and TOF on PET/CT image quality and determined the reconstruction parameters by phantom studies. We then also evaluated the impact of PSF and TOF on clinical images based on the results of the phantom studies. We found that the combination of PSF and TOF improved the SNR, contrast, and $\mathrm{CV}$ of the PET images in both phantom and clinical studies.

The CV of the images reconstructed by algorithms with PSF were superior to those without PSF. Furthermore, the $\mathrm{SNR}_{10} \mathrm{~mm}$ and contrast with PSF were superior to those without PSF at later iterations, as is consistent with previous studies reporting that the PSF algorithms converge more slowly than comparable non-PSF algorithms. This finding suggests that the PSF correction was mainly responsible for the improvement in image quality in this study. Contrast and CV increased when the number of iterations increased. A compromise trade-off needs to be found between the 2 conflicting performance parameters of high contrast and low CV. On the other hand, PET image reconstruction with PSF showed a more uniform background than did the images obtained using algorithms without PSF. The PSF algorithm, which includes additional information about the object to be recovered, slows iterative convergence. Thus, the PSF clearly decreased the noise compared with other algorithms at the same iteration numbers $(18,26)$. Furthermore, the highest $\mathrm{SNR}_{10} \mathrm{~mm}$ was obtained at iteration 3 in algorithms with PSF.

The TOF also improved the SNR in both phantom and clinical studies. According to the results of phantom 
studies, TOF improved the $\mathrm{SNR}_{10} \mathrm{~mm}$ and contrast. However, if we used the same iteration number, TOF did not seem to improve the noise. In the OSEM+TOF model, the contrast reached a maximum and converged uniformly with fewer iterations. Thus, it is acceptable to decrease the number of iterations for algorithms with TOF compared with those using conventional OSEM alone. TOF has been reported to improve the contrast of a small hot sphere and lead to a higher contrast recovery at a matched noise level, with faster and more uniform convergence $(18,20)$.

A combination of the PSF and TOF algorithm provided the best image quality among the reconstruction algorithms using either PSF or TOF (27). Both PSF and TOF greatly improved image quality: not only in the phantom study but also in the clinical study. In our phantom study, the PSF mainly improved the noise whereas TOF mainly increased the contrast at the same iteration number. As previously discussed, TOF leads to a faster convergence at a matched noise level with uniform convergence. On the other hand, PSF improved contrast and noise, although it slowed the iterative convergence (26). The balance of contrast recovery and noise is affected by the number of iterations (28). Therefore, it is important to examine the optimal reconstruction parameters instead of fixed conventional parameters. Several approaches have been proposed to determine the optimal number of iterations. Some make it possible to compare images using SNR or contrast recovery. Others can compare images at a given noise level, at either the same or different iterations for TOF and non-TOF. Optimization of the reconstruction parameters is essential to make the best of PSF or TOF.

Increasing the FWHM of the gaussian filter generally decreases the noise, and the contrast of the hot sphere is also decreased. The degree of improvement in image quality by both PSF and TOF was prominent with a small-FWHM gaussian filter, though the relative changes in contrast and CV differ for the different reconstruction algorithms. Thus, SNR may be affected differently as the filter strength is increased. In our study, the optimal FWHM of the gaussian filter was recommended to be either no filtering or less than $2 \mathrm{~mm}$ for algorithms with PSF and 4-6 $\mathrm{mm}$ for those without PSF. The clinically optimal FWHM for both PSF and TOF must be further examined to obtain high-diagnostic-quality PET images.

NEC has been widely used as an index to evaluate the quality of the acquired PET data (29). NEC is known to be directly proportional to the SNR of the acquired data and to depend on the injected dose and patients' BMI (30,31). The quality of clinical PET images is strongly influenced by patient weight, acquisition time, and injected dose $(32,33)$. In our study, the reconstruction algorithms with PSF+TOF improved the $\mathrm{SNR}_{\text {liver }}$. Therefore, the combination of PSF and TOF has the potential to maintain image quality for lower-activity objects and a shorter acquisition time. Our results showed that the $\mathrm{SNR}_{\text {liver }}$ of the low$\mathrm{NEC}_{\text {liver }}$ group with OSEM+PSF+TOF was comparable

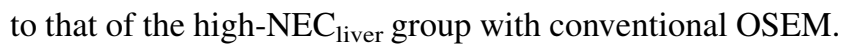
However, the degree of $\mathrm{SNR}_{\text {liver }}$ improvement brought about by the combination of PSF and TOF was not related to the $\mathrm{NEC}_{\text {liver }}$ value. Although TOF is considered to improve image quality, the degree of improvement has been reported to be influenced by patient size (22). Previous studies showed a correlation between BMI and SNR improvement by TOF (18). The maximum BMI of the examined patients was 31.6 (range, 16.0-31.6) in our study, whereas that in a previous report was 43 (range, 16-43) (18). The relationship between TOF and BMI may not have been observed in our study because of the narrow range of BMI. Therefore, a further study to directly compare the relationship between the TOF improvement and BMI, including patients with higher BMIs, is necessary.

This study had some limitations. First, we did not evaluate the detectability of lesions. Although our results indicated that the combination of PSF and TOF improved the SNR in normal liver, from a diagnostic aspect, detectability of small lesions is considered to be important. Furthermore, contrast, noise, and SNR were changed depending on the reconstruction algorithms and parameters used in our phantom study. Therefore, the standardized uptake value is also considered to be changed. A further study is required to examine the detectability of small lesions in relation to the standardized uptake value of lesions. Second, we determined the reconstruction parameters based on simple physical assessments, because such methods can easily be performed at many clinical institutions. Further examinations to evaluate more physically specialized features using more rigorous methods may be recommended $(19,21)$.

\section{CONCLUSION}

Both PSF and TOF provided a significant improvement in the quality of clinical PET images, and our findings indicate that optimization of the reconstruction parameters is necessary to obtain the best performance for PSF or TOF. A combination of PSF and TOF has the potential to obtain good image quality for a lower activity or a shorter acquisition time, thus improving patient comfort and reducing the radiation burden.

\section{DISCLOSURE STATEMENT}

The costs of publication of this article were defrayed in part by the payment of page charges. Therefore, and solely to indicate this fact, this article is hereby marked "advertisement" in accordance with 18 USC section 1734.

\section{ACKNOWLEDGMENTS}

We thank the staff of the Department of Clinical Radiology and Medical Technology at Kyushu University Hospital for their valuable clinical support. This study was supported in part by Grant-in-Aid 22611012 for Scientific 
Research (C) from the Japan Society for the Promotion of Science KAKENHI. No other potential conflict of interest relevant to this article was reported.

\section{REFERENCES}

1. Delbeke D. Oncological applications of FDG PET imaging: brain tumors, colorectal cancer, lymphoma and melanoma. J Nucl Med. 1999;40:591603.

2. Hicks RJ, Kalff V, MacManus MP, et al. The utility of ${ }^{18}$ F-FDG PET for suspected recurrent non-small cell lung cancer after potentially curative therapy: impact on management and prognostic stratification. J Nucl Med. 2001;42:16051613.

3. Oyen WJ, Bussink J, Verhagen AF, Corstens FH, Bootsma GP. Role of FDG-PET in the diagnosis and management of lung cancer. Expert Rev Anticancer Ther. 2004;4:561-567.

4. Vansteenkiste J, Fischer BM, Dooms C, Mortensen J. Positron-emission tomography in prognostic and therapeutic assessment of lung cancer: systematic review. Lancet Oncol. 2004;5:531-540.

5. Fletcher JW, Djulbegovic B, Soares HP, et al. Recommendations on the use of ${ }^{18}$ F-FDG PET in oncology. J Nucl Med. 2009;49:480-508.

6. Okubo M, Nishimura Y, Nakamatsu K, et al. Radiation treatment planning using positron emission and computed tomography for lung and pharyngeal cancers: a multiple-threshold method for $\left[{ }^{18} \mathrm{~F}\right]$ fluoro-2-deoxyglucose activity. Int J Radiat Oncol Biol Phys. 2010;77:350-356.

7. Halpern BS, Dahlbom M, Quon A, et al. Impact of patient weight and emission scan duration on PET/CT image quality and lesion detectability. J Nucl Med. 2004;45:797-801.

8. Tong S, Alessio AM, Kinahan PE. Noise and signal properties in PSF-based fully 3D PET image reconstruction: an experimental evaluation. Phys Med Biol. 2010;55:1453-1473.

9. Alessio AM, Stearns CW, Tong S, et al. Application and evaluation of a measured spatially variant system model for PET image reconstruction. IEEE Trans Med Imaging. 2010;29:938-949.

10. Surti S, Karp JS, Popescu LM, Daube-Witherspoon ME, Werner M. Investigation of time-of-flight benefit for fully 3-D PET. IEEE Trans Med Imaging. 2006; 25:529-538.

11. Panin VY, Kehren F, Michel C, Casey M. Fully 3-D PET reconstruction with system matrix derived from point source measurements. IEEE Trans Med Imaging. 2006;25:907-921.

12. Anger HO. Survey of radioisotope cameras. ISA Trans. 1966;5:311-334.

13. Brownell GL, Burnham CA, Wilensky S, Aronow S, Kazemi H, Streider D. New developments in positron scintigraphy and the application of cyclotron produced positron emitters. In: Medical Radioisotope Scintigraphy. Vol 1. Vienna, Austria: International Atomic Energy Agency; 1969:163-176.

14. Allemand R, Gresset C, Vacher J. Potential advantages of a cesium fluoride scintillator for a time-of-flight positron camera. J Nucl Med. 1980;21:153-155.
15. Moses WW, Derenzo SE. Prospects for time-of-flight PET using LSO scintillator. IEEE Trans Nucl Sci. 1999;46:474-478.

16. Melcher CL, Schweither JS. Cerium-doped lutetium oxyorthosilicate: a fast, efficient new scintillator. IEEE Trans Nucl Sci. 1992;39:502-505.

17. Daghighiam F, Shenderov P, Pentlow KS, et al. Evaluation of cerium doped lutetium oxyorthosilicate (LSO) scintillation crystal for PET. IEEE Trans Nucl Sci. 1993;40:1045-1047.

18. Lois C, Jakoby BW, Long MJ, et al. An assessment of the impact of incorporating time-of-flight information into clinical PET/CT imaging. J Nucl Med. 2010;51:237-245.

19. Kadrmas DJ, Casey ME, Conti M, Jakoby BW, Lois C, Townsend DW. Impact of time-of-flight on PET tumor detection. J Nucl Med. 2009;50:1315-1323.

20. Karp JS, Surti S, Daube-Witherspoon ME, Muehllehner G. Benefit of time-offlight in PET: experimental and clinical results. J Nucl Med. 2008;49:462-470.

21. El Fakhri G, Surti S, Trott CM, Scheuermann J, Karp JS. Improvement in lesion detection with whole-body oncologic time-of-flight PET. J Nucl Med. 2011; 52:347-353.

22. Surti S, Scheuermann J, El Fakhri G, et al. Impact of time-of-flight PET on whole-body oncologic studies: a human observer lesion detection and localization study. J Nucl Med. 2011;52:712-719.

23. Fukukita H, Senda M, Terauchi T, et al. Japanese guideline for the oncology FDG-PET/CT data acquisition protocol: synopsis of version 1.0. Ann Nucl Med. 2010;24:325-334.

24. Paquet N, Albert A, Foidart J, Hustinx R. Within-patient variability of ${ }^{18}$ F-FDG: standardized uptake values in normal tissues. J Nucl Med. 2004;45:784-788.

25. Badawi RD, Dahlbom M. NEC: some coincidences are more equivalent than others. J Nucl Med. 2005;46:1767-1768.

26. Thielemans K, Asma E, Ahn S, et al. Impact of PSF modeling on the convergence rate and edge behavior of EM images in PET. IEEE Nucl Sci Symp Conf Rec. 2010;3267-3272.

27. Bettinardi V, Presotto L, Rapisarda E, Picchio M, Gianolli L, Gilardi MC. Physical performance of the new hybrid PET/CT Discovery-690. Med Phys. 2011;38:5394-5411.

28. Conti M. Focus on time-of-flight PET: the benefits of improved time resolution. Eur J Nucl Med Mol Imaging. 2011;38:1147-1157.

29. Nagaki A, Onoguchi M, Matsutomo N. Patient weight-based acquisition protocols to optimize ${ }^{18}$ F-FDG PET/CT image quality. J Nucl Med Technol. 2011;39:72-76.

30. Strother SC, Casey ME, Hoffman EJ. Measuring PET scanner sensitivity: relating countrates to image signal-to-noise ratios using noise equivalent counts. IEEE Trans Nucl Sci. 1990;37:783-788.

31. Lartizien C, Comtat C, Kinahan PE, Ferreira N, Bendriem B, Trebossen R. Optimization of injected dose based on noise equivalent count rates for 2- and 3-dimensional whole-body PET. J Nucl Med. 2002;43:1268-1278.

32. Masuda Y, Kondo C, Matsuo Y, Uetani M, Kusakabe K. Comparison of imaging protocols for ${ }^{18} \mathrm{~F}$-FDG PET/CT in overweight patients: optimizing scan duration versus administered dose. J Nucl Med. 2009;50:844-848.

33. Tatsumi M, Clark PA, Nakamoto Y, Wahl RL. Impact of body habitus on quantitative and qualitative image quality in whole-body FDG-PET. Eur J Nucl Med Mol Imaging. 2003;30:40-45. 